\title{
Slug overexpression induces stemness and promotes hepatocellular carcinoma cell invasion and metastasis
}

\author{
YU SUN*, GUO-DONG SONG* ${ }^{*}$, NING SUN, JIAN-QIU CHEN and SHAO-SHI YANG \\ Department of Surgery, The Second Hospital of Tianjin Medical University, Hexi, Tianjin 300211, P.R. China
}

Received July 29, 2013; Accepted February 4, 2014

DOI: $10.3892 / 01.2014 .2037$

\begin{abstract}
Detection of metastasis of hepatocellular carcinoma (HCC) is crucial for early diagnosis. Epithelial-mesenchymal transition (EMT) is a common event in the metastasis of tumor cells. Slug and Snail are homologous proteins, which play an important role in EMT. The present study aimed to investigate whether Slug and Snail overexpression is associated with the invasiveness of HCC in vitro and in vivo. Invasion, colony formation and wound healing assays, as well as flow cytometry analysis, were performed to examine the invasiveness and proliferation capabilities of HepG2 cells following transfection with cNDA or the siRNA of Slug or Snail. The effects of Slug on HCC in vivo were examined using a xenograft model. Slug upregulation increased the percentage of cluster of differentiation (CD) $133^{+}$cells among HepG2 cells, and induced cell invasion and proliferation; whereas Snail upregulation did not affect the cells in vitro. The Slug overexpression group exhibited the highest rate of tumor growth compared with the Snail overexpression and control groups in vivo. These findings demonstrated that Slug increases the percentage of CD133 ${ }^{+}$ cells, promotes the clonigenicity of HCC cells and induces a stronger stemness in Slug-overexpressing cells. These changes activate dormant developmental pathways in invading tumor cells. Thus, Slug may serve as a novel target for HCC prognosis and therapy.
\end{abstract}

\section{Introduction}

Hepatocellular carcinoma (HCC) is the fifth most common malignancy worldwide and is the third leading cause of cancer-related mortality in China (1). HCC is a rapidly growing tumor associated with a propensity for vascular

Correspondence to: Professor Shao-Shi Yang, Department of Surgery, The Second Hospital of Tianjin Medical University, 23 Pingjiang Road, Hexi, Tianjin 300211, P.R. China

E-mail: ssyang009@163.com

*Contributed equally

Key words: hepatocellular carcinoma, epithelial-mesenchymal transition, Slug, stemness invasion and metastasis, which results in poor cancer prognoses (2). Considering that methods for early detection of HCC are currently unavailable, the majority of patients present with peritoneal dissemination and distant metastasis at the time of diagnosis (3). Thus, treatment is often unsuccessful and overall survival time is short. Consequently, identifying invasion-related molecules associated with the early and rapid spread of HCC is a focus of investigation.

Invasion and metastasis are the biological hallmarks of malignancy (4). The molecular mechanisms underlying invasion and metastasis are an important area of investigation. Epithelial-mesenchymal transition (EMT) is a common event in the plasticity of tumor cells $(5,6)$. As invasion proceeds, epithelial cell layers lose polarity and cell-cell interaction, which ultimately results in the complex remodeling of the cytoskeleton. EMT is an embryonic trait by which cells adopt a phenotype that is more suitable to migration and invasion (7). A number of molecules associated with tumor invasion and EMT in HCC have been reported, including Twist, Snail and Slug (8-10). However, the molecular changes associated with the metastatic ability in HCC progression have not been clearly determined.

\section{Materials and methods}

Cell culture and transfection. The HepG2 cell line was obtained from the American Type Culture Collection (Rockville, MD, USA). The cells were cultured in Dulbecco's modified Eagle's medium (DMEM) supplemented with $10 \%$ fetal bovine serum (FBS; Invitrogen Life Technologies, Carlsbad, CA, USA). pcDNA3.1/Slug, pcDNA3.1/Snail and pcDNA3.1 empty vector (Clontech, Palo Alto, CA, USA) were transfected into the cells using polyethylenimine (cat no. 23966; Polysciences, Inc., Warrington, PA, USA).

Expression plasmids. Full-length Snail and Slug cDNA were generated using normal human embryo total cDNA, cleaved with $X h o I / E c o R I$ and subcloned into pcDNA3.1 vectors. The resulting constructs were confirmed by DNA sequencing. The pcDNA3.1 empty vector was used as a cDNA control. Small interfering (si)RNA-coding oligonucleotides against human Snail and Slug were designed and verified for specificity for Snail and Slug. The Snail siRNA targeting sequence was AAGCTGAGCAAGATTCAGACC and the Slug siRNA targeting sequence was CAGGACCTCGCCGCTGCAGAC 
(siB-cell lymphoma 2 nucleotides 200-221) (11). The U6 promoter with a Snail or Slug siRNA insert was subcloned into pRNA-U6-Neo. A non-silencing siRNA sequence (target sequence, AATTCTCCGAACGTGTCACGT) was used as the negative control.

Invasion and wound healing assays. The cell migration assay was performed using Transwell cell culture inserts (Invitrogen Life Technologies). The transfected cells were maintained for $48 \mathrm{~h}$ and allowed to migrate for an additional $24 \mathrm{~h}$. The passaged cells were stained with crystal violet solution and absorbance was measured at $595 \mathrm{~nm}$. In the wound healing assays, cell motility was assessed by measuring the movement of cells towards the scratch. The speed of wound closure was monitored after 12 and $24 \mathrm{~h}$ by measuring the ratio of the distance of the wound at $0 \mathrm{~h}$. Each experiment was performed in triplicate.

Colony formation assay. The control and transfected cells were seeded into six-well plates at a density of 1,000 cells/well. After two weeks, the clones were fixed in methanol and stained with 2\% Giemsa solution (Merck KGaA, Darmstadt, Germany) for $10 \mathrm{~min}$.

Flow cytometry analysis. Following treatment, the cells were fixed in $75 \%$ ethanol and indirectly labeled by incubation with the primary anti-SNAI1 rabbit polyclonal (ab82846; Abcam, Cambridge, MA, USA), anti-SNAI2 mouse monoclonal (ab51772; Abcam), anti-E-cadherin rabbit polyclonal (sc-7870; Santa Cruz Biotechnology, Inc., Santa Cruz, CA, USA), anti-vimentin mouse monoclonal (ab20346; Abcam) and anti-CD133 mouse monoclonal (130080801; Miltenyi Biotech, San Diego, CA, USA) and the secondary antibodies; goat anti-mouse polyclonal IgG horse radish peroxidase (HRP) conjugated antibody (sc-2005, Santa Cruz Biotechnology, Inc.) and goat anti-rabbit polyclonal IgG-HRP conjugated antibody (sc-2004, Santa Cruz Biotechnology, Inc.). The percentage of CD133-positive cells was identified by using flow cytometric analyses with the CD133 monocolonal antibody directly conjugated with phycoerythrin (Miltenyi Biotec, Auburn, CA, USA). All cells were stained and incubated with a CD133-phycoerythrin antibody. A C6 flow cytometer (BD Accuri $^{\mathrm{TM}}$, Franklin Lakes, NJ, USA) was used to determine the percent of CD133-positive cells.

Western blot analysis. Whole cell lysates were resolved by sodium dodecyl sulfate-polyacrylamide gel electrophoresis (Invitrogen Life Technologies) and transferred onto polyvinylidene difluoride membranes (Millipore, Billerica, MA, USA). Blots were blocked and incubated with the mouse monoclonal antibody (anti-SNAI2; ab51772; Abcam) followed by incubation with a secondary antibody (1:2,000; Santa Cruz Biotechnology, Inc.). Blots were developed using an enhanced chemiluminescence detection kit (Amersham Pharmacia Biotech, Inc., Piscataway, NJ, USA). Monoclonal $\beta$-actin antibody (1:200; Santa Cruz Biotechnology, Inc.) was used for protein loading analyses.

Murine xenograft model. Female BALB/c-null mice (age, six weeks) were obtained from the National Institutes of Health
(Bethesda, MD, USA) and housed in the animal facilities at the Tianjin Medical University (Tianjin, China), as approved by the Institutional Animal Care and Use Committee. The HepG2 cells $\left(10^{7}\right.$ cells $\left./ \mathrm{ml}\right)$ were mixed with Matrigel (BD Biosciences, Franklin Lakes, NJ, USA) and subcutaneously injected into the backs of the nude mice $(0.1 \mathrm{ml} / \mathrm{mouse})$. The mice were monitored and tumor sizes were measured daily using a caliper for 25 days. The experiments were terminated after 25 days due to the tendency of HepG2 cells to become necrotic and form skin ulcers. The mice were sacrificed by $\mathrm{CO}_{2}$ asphyxiation following observations. The tumors were harvested and stored at $-80^{\circ} \mathrm{C}$ for subsequent tests.

Sulfrodamine B (SRB) cell proliferation assay. Cells were seeded in a 96-well plate (Corning Inc., Corning, NY, USA) at a final concentration of 5000 cells per well follwoing transfection and maintained in DMEM (Gibco-Brl, Grand Island, NY, USA) containing $10 \%$ FBS (Gibco-Brl) at $37^{\circ} \mathrm{C}$ in a humidified atmosphere containing $5 \% \mathrm{CO}_{2}$. Cell proliferation was analyzed every 8 hours. Initially the cells were washed with distilled water after being fixed with trichloroacetic acid, and were subsequently stained for $10 \mathrm{~min}$ with sulforhodamine B (Sigma-Aldrich, St. Louis, MO, USA) dissolved in $1.0 \%$ acetic acid (Sigma-Aldrich). The plates were washed with $1.0 \%$ acetic acid and allowed to air dry. Finally, $150 \mu 1$ of $10 \mathrm{mM}$ Tris base (Sigma-Aldrich) was added to each well in order to solubilize the dye, and the absorbance at $490 \mathrm{~nm}$ was determined with the use of a microplate reader (Synergy H4, Bio-Tek, Winooski, VT, USA).

Statistical analysis. All the data were evaluated using SPSS software, version 11.5 (SPSS, Inc., Chicago, IL, USA) and analyzed using the Student's t-test and analysis of variance. $\mathrm{P}<0.05$ was considered to indicate a statistically significant difference. The significant groups are marked with asterisks as shown in the figures.

\section{Results}

Slug upregulation increases HepG2 cell proliferation in vitro. The effects of Slug expression on cell invasion, migration and clone formation were investigated. E-cadherin expression is frequently associated with metastatic ovarian carcinoma (12). Metastasis is associated with cell migration and invasion, and the underlying mechanisms are similar to those of EMT. The invasiveness and migration capability of HepG2 cells in the Slug and Snail transfection and knockdown groups were examined. Western blot analysis revealed a significant difference in the ectopic transfection groups compared with the control group. Slug and Snail were upregulated in the overexpressed group and were downregulated in the siRNA group (Fig. 1A). The sulforhodamine B protein assay was used to measure cell numbers and a significant difference in cell proliferation was observed between the Slug overexpression and knockdown groups. The cells exhibited a significant decrease in cell proliferation in the Slug knockdown group, whereas cell proliferation was significantly increased in the overexpressed group compared with the control group $(\mathrm{P}<0.01)$. Snail expression did not significantly affect cell proliferation $(\mathrm{P}>0.01)$. 


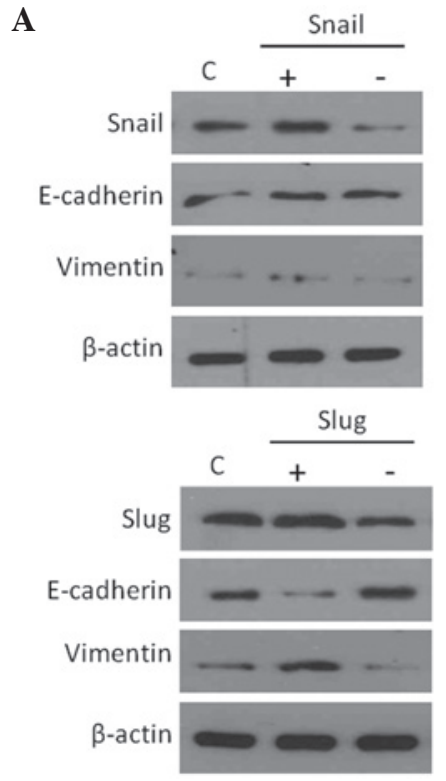

B
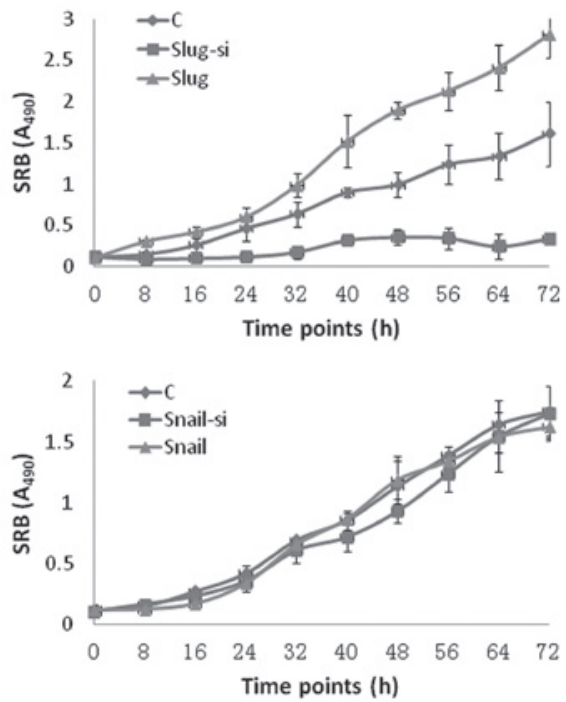

Figure 1. Slug expression promotes HCC cell proliferation. The HepG2 cells were transfected with Slug and Snail expression plasmids and small interfering RNA plasmids. (A) Western blot analysis and (B) the SRB protein assay analyzed the correlation of invasion-related protein levels with HepG2 cell proliferation. HCC, hepatocellular carcinoma; C, control; SRB, sulforhodamine B; si, small interfering (gene knockdown).

A

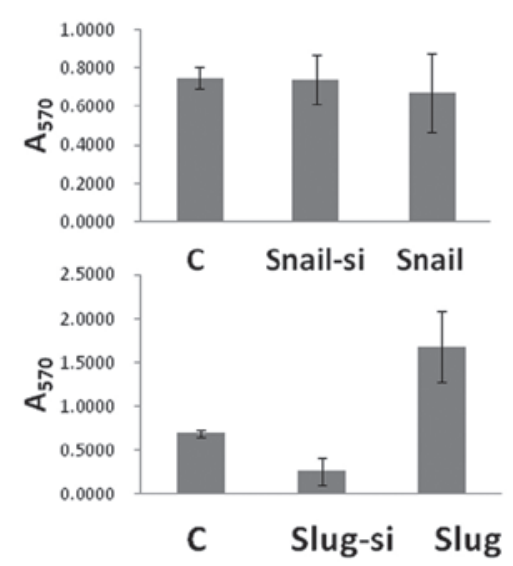

B
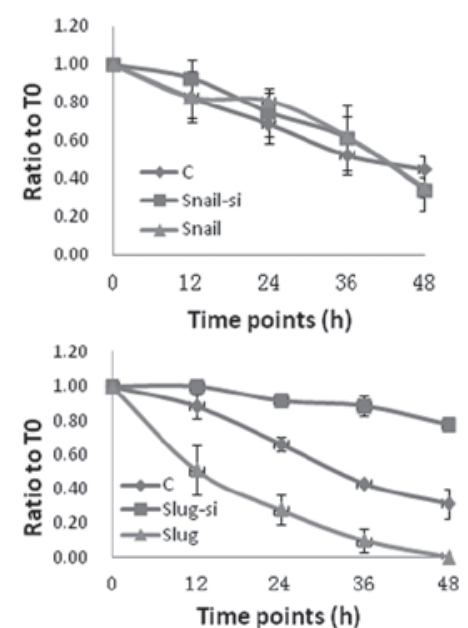

C

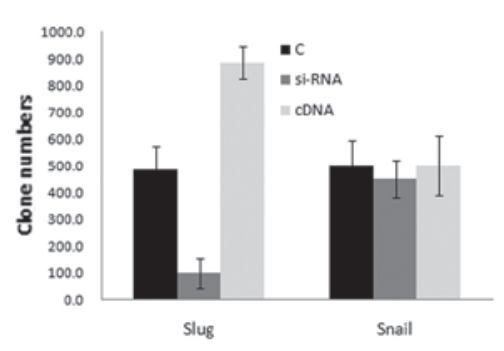

Figure 2. Slug expression promotes HepG2 cell invasion, migration and clone formation. The HepG2 cells were transfected with Slug and Snail expression plasmids and si RNA plasmids. (A) Invasion and (B) wound healing assays tested the migration capability and invasiveness of HepG2 cells. (C) A colony formation assay analyzed the effects of Slug and Snail on the clone formation of HepG2 cells. C, control; siRNA, small interfering RNA.

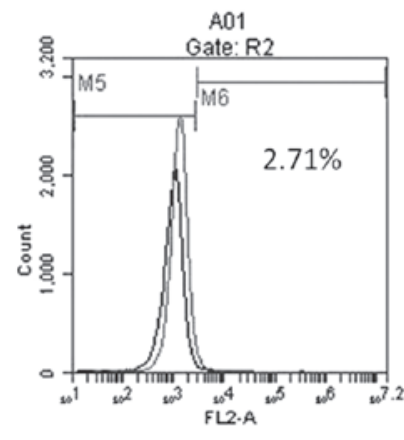

Control

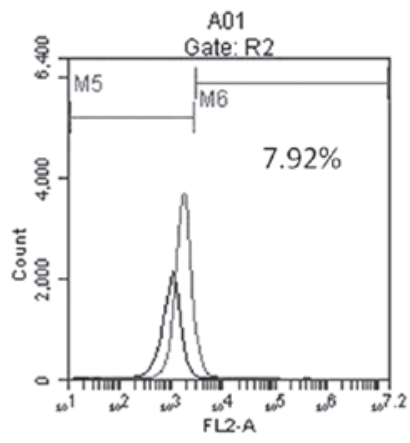

Snail

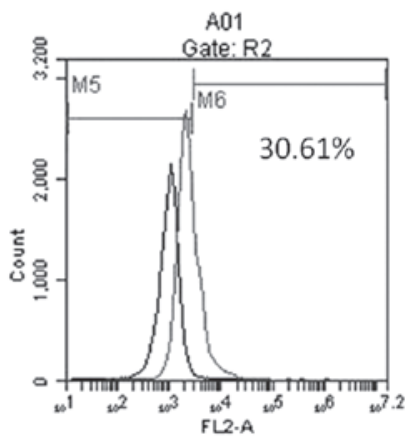

Slug

Figure 3. Slug expression increases the number of $\mathrm{CD} 133^{+}$cells among the HepG2 cells. Flow cytometry analyzed the percentage of $\mathrm{CD} 133^{+}$cells among HepG2 cells transfected with Slug and Snail cDNA, and in the controls. CD, cluster of differentiation. 
A

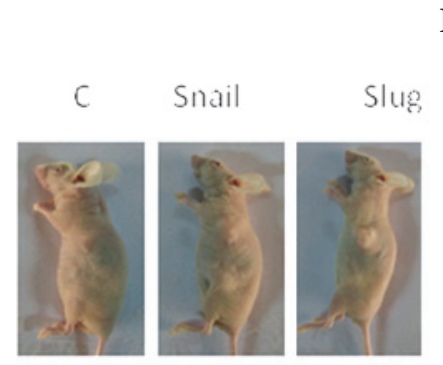

C

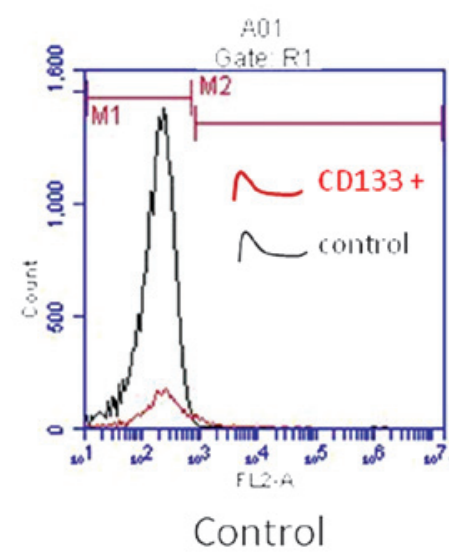

B
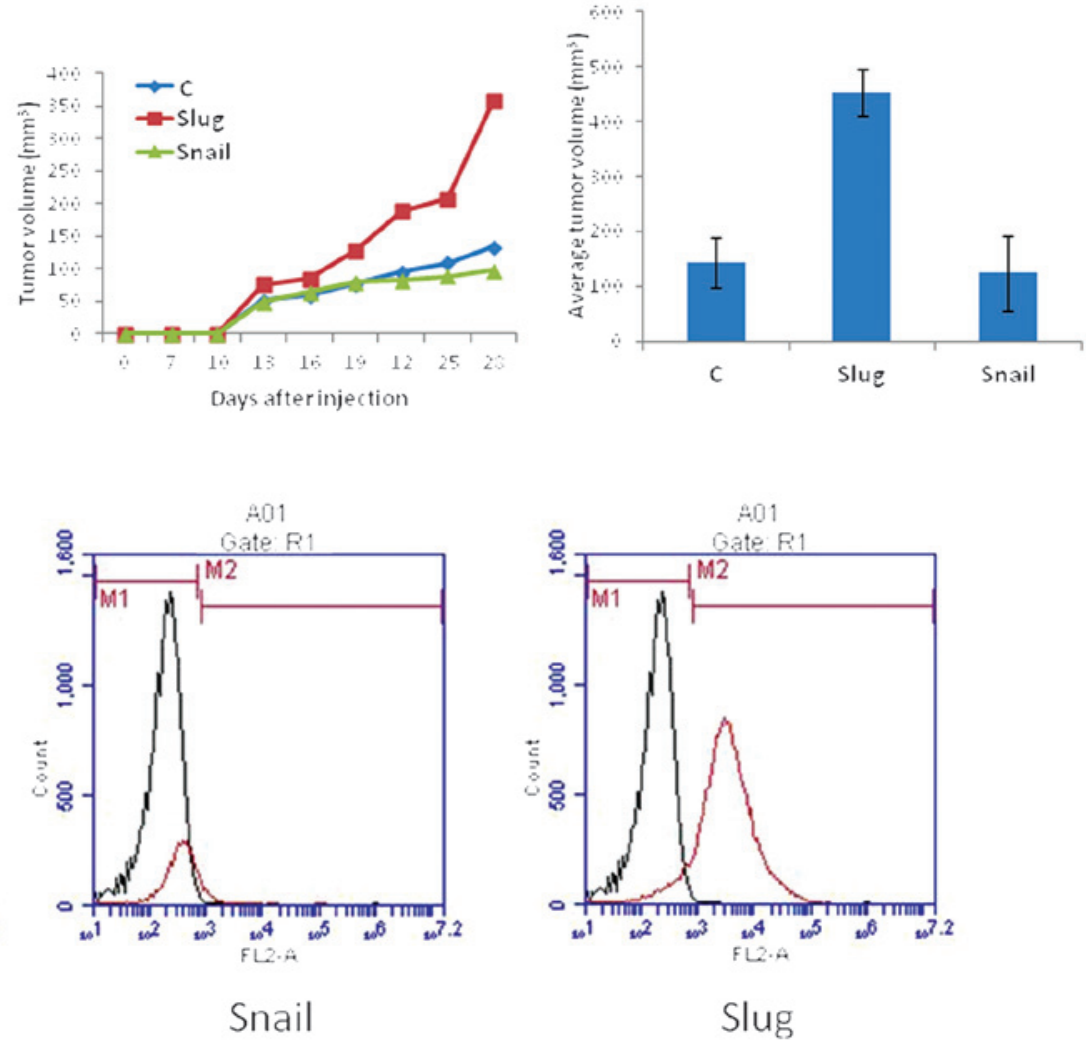

Figure 4. Slug expression increases tumor growth and the percentage of CD133+ cells in vivo. The HepG2 cells transfected with Slug and Snail cDNA were injected into nude mice. (A and B) The tumor volume was examined and recorded, and (C) the percentage of CD133 ${ }^{+}$cells was determined by flow cytometry. $\mathrm{C}$, control; $\mathrm{CD}$, cluster of differentiation.

Slug upregulation increases HCC cell invasion, migration and clone formation in vitro. The HepG2 cell cultures were analyzed for functional changes in migration, invasion and clone formation following transfection with Slug and Snail, separately. Compared with the transfection and control groups, the Slug overexpression group exhibited a significantly increased activity in the migration, invasion and clonigenicity assays (Fig. 2). The effects of non-uniform transfection efficiency were minimized by selecting Slug siRNA-transfected cells for slug knockdown, and the Slug knockdown group revealed decreased activity in the migration, invasion and clonigenicity assays compared with the control group. Notably, Snail, which shares structural homology with Slug, did not promote cell migration.

Slug upregulation increases the percentage of cluster of differentiation $(C D) 133^{+}$cells in HCC cells. Stem cells play an important role in tumor cell plasticity and proliferation during tumor metastasis, and are strongly associated with EMT. In this study, flow cytometry examined the percentage of $\mathrm{CD} 133^{+}$cells in the Slug overexpression and knockdown groups. The CD133+ cells accounted for $47.5 \%$ of the cells in the Slug overexpression group, $8.7 \%$ in the control transfection group and $0.4 \%$ in the Slug knockdown group (Fig. 3). No significant difference in the percentage of $\mathrm{CD} 133^{+}$cells was observed between the Snail overexpression, Snail knockdown and control groups. These findings suggest that synergism between Slug and $\mathrm{CD}_{133^{+}}$cells increases cell proliferation, migration and invasion.
Coexpression of CD133 and Slug correlates with tumor proliferation. A murine xenograft model was used to examine the in vivo effects of Slug and Snail expression on tumor development. The HepG2 cells were utilized to establish xenografts in nude mice. Nodule formation and growth (volume) were monitored over 25 days. The Slug overexpression group displayed the highest rate of tumor growth compared with the Snail overexpression (60\%) and control (50\%) groups (Fig. 4). The cells were collected from the tumor tissues and analyzed using flow cytometry. The findings demonstrated a significant increase in $\mathrm{CD}_{133^{+}}$cells in the Slug overexpression group compared with the control group, but no significant difference was observed between the Snail overexpression and the control groups (Fig. 4).

\section{Discussion}

HCC invasiveness is a key step that results in metastasis and a poor prognosis $(1,11)$; therefore, the underlying molecular mechanisms are a focus of investigation. EMT plays an important role in the development of tissues during embryogenesis (13). However, similar cell changes are recapitulated during pathological processes, such as in cancer progression. Previous studies on EMT have focused on cancer cell invasion and metastasis $(6,14)$. Several developmental genes that induce EMT act as E-cadherin repressors. The first of these genes is the Snail family of zinc-finger protein transcription factors, which is a DNA-binding factor family that recognizes E-box motifs in target promoters, such as E-cadherin. Slug is homologous to 
Snail and was the first transcriptional repressor of E-cadherin to be described along with other non-Snail transcriptional repressors of E-cadherin [such as, E47, delta-crystallin/E2-box factor 1/zinc finger E-Box-binding homeobox (Zeb) 1 and Smad-interacting protein 1/Zeb2] (15-18). These repressors are tightly regulated at the transcriptional level and/or by subcellular localization. Insights into the underlying mechanisms of EMT regulation may provide novel chemotherapeutic and antifibrotic therapies.

Tumor progression and invasion are complex biological processes that involve the remodeling of stromal tissue by invading cells $(12,19-21)$. Slug and Snail are members of an evolutionarily conserved family of zinc-finger transcription factors. Slug and Snail are expressed in the intermediate mesoderm and the metanephric mesenchyme during renal development, and are downregulated prior to epithelial differentiation $(22,23)$. The kidneys developed normally in mice with a loss-of-function mutation in Snail, which suggests the functional redundancy of Snail and Slug $(24,25)$. However, the E-cadherin repressors, Snail, Slug and E47, produce different genetic profiles when overexpressed in ovarian tumor cells, suggesting differential regulation of these transcription factors $(8,26-28)$. In the present study, the constitutive expression of Slug increased invasion by inducing EMT and the results obtained following gene knockdown were consistent with those of a previous study (21). Our findings suggest that Slug induces EMT, increases the percentage of $\mathrm{CD} 33^{+}$cells, promotes the clonigenicity of HCC cells and induces a stronger stemness. These changes activate dormant developmental pathways in invading tumor cells; therefore, suppressing invasion-related molecules, such as Slug, may present an important mechanism to suppress metastasis. Furthermore, increased Snail levels did not significantly affect HCC cell proliferation, migration, invasion, clonigenicity or the number of $\mathrm{CD} 133^{+}$cells. Thus, Snail proteins may have a polarizing effect on HCC tissue growth.

In conclusion, our findings demonstrated that Slug upregulation increased the number of $\mathrm{CD}_{133^{+}}$cells, which is important for EMT and proliferation of ovarian cancer cells. Therefore, Slug may be a potential new target for preventing tumor invasion and metastasis.

\section{References}

1. Yang MH, Chen CL, Chau GY, et al: Comprehensive analysis of the independent effect of twist and snail in promoting metastasis of hepatocellular carcinoma. Hepatology 50: 1464-1474, 2009.

2. Hanazaki K, Matsushita A, Nakagawa K, Misawa R and Amano J: Risk factors of long-term survival and recurrence after curative resection of hepatocellular carcinoma. Hepatogastroenterology 52: 552-557, 2005.

3. Yeoman AD, Al-Chalabi T, Karani JB, et al: Evaluation of risk factors in the development of hepatocellular carcinoma in autoimmune hepatitis: Implications for follow-up and screening. Hepatology 48: 863-870, 2008.

4. Hanazaki K, Matsushita A, Nakagawa K, Misawa R and Amano J: Risk factors of intrahepatic recurrence after curative resection of hepatocellular carcinoma. Hepatogastroenterology 52: 580-586, 2005.
5. Savagner P: The epithelial-mesenchymal transition (EMT) phenomenon. Ann Oncol 21 (Suppl 7): vii89-vii92, 2010.

6. Bacac M and Stamenkovic I: Metastatic cancer cell. Annu Rev Pathol 3: 221-247, 2008.

7. Kang Y and Massagué J: Epithelial-mesenchymal transitions: twist in development and metastasis. Cell 118: 277-279, 2004.

8. Smit MA, Geiger TR, Song JY, Gitelman I and Peeper DS: A Twist-Snail axis critical for TrkB-induced epithelial-mesenchymal transition-like transformation, anoikis resistance, and metastasis. Mol Cell Biol 29: 3722-3737, 2009.

9. Hotz B, Arndt M, Dullat S, Bhargava S, Buhr HJ and Hotz HG: Epithelial to mesenchymal transition: expression of the regulators snail, slug, and twist in pancreatic cancer. Clin Cancer Res 13: 4769-4776, 2007.

10. Šošić D, Richardson JA, Yu K, Ornitz DM and Olson EN: Twist regulates cytokine gene expression through a negative feedback loop that represses NF-kappaB activity. Cell 112: 169-180, 2003.

11. McCawley LJ and Matrisian LM: Tumor progression: defining the soil round the tumor seed. Curr Biol 11: R25-R27, 2001.

12. Sun T, Zhao N, Zhao XL, et al: Expression and functional significance of Twistl in hepatocellular carcinoma: its role in vasculogenic mimicry. Hepatology 51: 545-556, 2010.

13. Zhou C, Liu J, Tang Y and Liang X: Inflammation linking EMT and cancer stem cells. Oral Oncol 48: 1068-1075, 2012.

14. Childs G and Segall JE: Twists and turns of invasion. Nat Cell Biol 14: 337-339, 2012.

15. Firulli AB and Conway SJ: Phosphoregulation of Twist1 provides a mechanism of cell fate control. Curr Med Chem 15: 2641-2647, 2008.

16. Yang MH and Wu KJ: TWIST activation by hypoxia inducible factor-1 (HIF-1): implications in metastasis and development. Cell Cycle 7: 2090-2096,2008.

17. Martin A and Cano A: Tumorigenesis: Twist1 links EMT to self-renewal. Nat Cell Biol 12: 924-925, 2010.

18. Pietras K and Ostman A: Hallmarks of cancer: interactions with the tumor stroma. Exp Cell Res 316: 1324-1331, 2010.

19. Peng JY and Wang Y: Tumor stroma: A determinant role in local recurrence of rectal cancer patients receiving total mesorectal excision? Med Hypotheses 75: 442-444, 2010.

20. Erenpreisa J and Cragg MS: Cancer: a matter of life cycle? Cell Biol Int 31: 1507-1510, 2007.

21. Sun T, Sun BC, Zhao XL, et al: Promotion of tumor cell metastasis and vasculogenic mimicry by way of transcription coactivation by Bcl-2 and Twist1: a study of hepatocellular carcinoma. Hepatology 54: 1690-1706, 2011.

22. Timmerman LA, Grego-Bessa J, Raya A, et al: Notch promotes epithelial-mesenchymal transition during cardiac development and oncogenic transformation. Genes Dev 18: 99-115, 2004.

23. Alonso-Magdalena P, Brössner C, Reiner A, et al: A role for epithelial-mesenchymal transition in the etiology of benign prostatic hyperplasia. Proc Natl Acad Sci USA 106: 2859-2863, 2009.

24. Ikenouchi J, Matsuda M, Furuse M and Tsukita S: Regulation of tight junctions during the epithelium-mesenchyme transition: direct repression of the gene expression of claudins/occludin by Snail. J Cell Sci 116: 1959-1967, 2003.

25. Carver EA, Jiang R, Lan Y, Oram KF and Gridley T: The mouse snail gene encodes a key regulator of the epithelial-mesenchymal transition. Mol Cell Biol 21: 8184-8188, 2001.

26. Smith JP, Pozzi A, Dhawan P, Singh AB and Harris RC: Soluble HB-EGF induces epithelial-to-mesenchymal transition in inner medullary collecting duct cells by upregulating Snail-2. Am J Physiol Renal Physiol 296: F957-F965, 2009.

27. Usami Y, Satake S, Nakayama F, et al: Snail-associated epithelial-mesenchymal transition promotes oesophageal squamous cell carcinoma motility and progression. J Pathol 215: 330-339, 2008.

28. Medici D, Hay ED and Olsen BR: Snail and Slug promote epithelial-mesenchymal transition through beta-catenin-T-cell factor-4-dependent expression of transforming growth factor-beta3. Mol Biol Cell 19: 4875-4887, 2008. 